\title{
Fuzzification of Linear Combination Based on Marshall-Olkin Bivariate Exponential Distribution
}

\author{
Yangjing Chong, Fangfang Guo, Wenyuan Sun* \\ Mathematics Department, Yanbian University, Yanji, P. R. China \\ Email address: \\ 513603970@qq.com (Yangjing Chong),1024821072@qq.com (Fangfang Guo),112277188@qq.com (Wenyuan Sun) \\ *Corresponding author
}

\section{To cite this article:}

Yangjing Chong, Fangfang Guo, Wenyuan Sun. Fuzzification of Linear Combination Based on Marshall-Olkin Bivariate Exponential Distribution. International Journal of Management and Fuzzy Systems. Vol. 3, No. 2, 2017, pp. 28-31. doi: 10.11648/j.ijmfs.20170302.12

Received: April 7, 2017; Accepted: May 16, 2017; Published: June 16, 2017

\begin{abstract}
The exponential distribution is applied to a very wide range of life analysis models, therefore, the research on it is of great significance in the practice of life. Based on the basic theory of general reliability and fuzzy reliability, on account of the Marshall-Olkin binary exponential distribution model, by establishing the fuzzy probability density function of the linear combination of two - dimensional random variables, this paper gives the fuzzy reliability function of the linear combination of the most widely used two - dimensional exponential distribution in reliability engineering.
\end{abstract}

Keywords: Fuzzy Reliability, Binary Exponential Distribution, Non-Independent, Linear Combination

\section{Introduction}

The exponential distribution has been widely used in reliability theory and life analysis because of its memoryless and other properties [1]. So far, the research on the distribution of one dollar index and its application has been quite rich [2]. The exponential distribution is applied to the failure distribution of a product with a constant failure rate, such as most electronic equipment.The exponential distribution can be used to describe the failure distribution of mechanical or electromechanical products with little influence on wearout failure during the service life. The exponential distribution can be used as the failure distribution of mechanical or electromechanical products for regular maintenance, repair or replacement of parts. The exponential distribution can be applied to the life and reliability analysis of a mechanical or electromechanical device or system consisting of a number of components with an initial failure rate of zero [3]. In recent years, a variety of literature has also proposed a variety of exponential distribution of two-dimensional extension [4], Zhang Ping [5] conducted a statistical analysis of various binary exponential distributions. Marshall-Olkin is the only multivariate exponential distribution with no memory. It can also be said that the Marshall-Olkin type binary exponential distribution is the only binary exponential distribution with exponential edge distribution and no memory [6]. For a given random variable
$\mathrm{X}, \mathrm{Y}$ that obeys the Marshall-Olkin two-dimensional exponential distribution, the distribution of this linear combination form of $\alpha X+\beta Y$ is significant in quality engineering and reliability engineering [7]. In reliability engineering, there will be a lot of uncertain information, The traditional reliability theory is based on the uncertainty of randomness. However, in practice, because the data we have insufficient information, or the presence of expert experience and subjective factors, for this kind of uncertain information processing, experience more difficult to apply the probability method, the application of fuzzy analysis method is more suitable to deal with [8]. The fuzzy reliability analysis method considers more factors than the classical reliability theory. It can be said that the fuzzy reliability theory is an extension and development of the traditional classical reliability theory. In the field of fuzzy reliability, Li Tingjie and $\mathrm{Gao} \mathrm{He}$ [9] give fuzzy reliability function of general variables if they are independent random variables. Guo Yunfei [10] gives the reliability function of two-dimensional random variables under non-independent conditions without fuzzy. Based on this consideration, this paper gives the fuzzy reliability function of random variable $Z=\alpha X+\beta Y$ under non - independent condition. 


\section{Pre-knowledge}

Definition 2.1 If the random variables $(X, Y)$ obey the
Marshall-Olkin binary exponential distribution model with parameters $\lambda_{1}, \lambda_{2}$ and $\lambda_{3}$, then the joint survival probability [11] is

$$
\bar{F}(x, y) \triangleq P(X \succ x, Y \succ y)=\exp \left\{-\lambda_{1} x-\lambda_{2} y-\lambda_{3} \max (x, y)\right\}, x \succ 0, y \succ 0
$$

Among this $\lambda_{1}, \lambda_{2}, \lambda_{3} \succ 0 \quad, \quad$ denoted as $(X, Y) \sim B V E\left(\lambda_{1}, \lambda_{2}, \lambda_{3}\right)$.

Definition 2.2 If the random variables $(X, Y)$ obey the Marshall-Olkin binary exponential distribution model with parameters $\lambda_{1}, \lambda_{2}$ and $\lambda_{3}$, according to the joint survival probability [12], it is true that marginal survival probabilities of $\mathrm{X}$ and $\mathrm{Y}$ are

$$
\begin{aligned}
& \bar{F}_{1}(x)=P\{X \succ x\}=\exp \left\{-\left(\lambda_{1}+\lambda_{3}\right) x\right\}, x \geq 0 \\
& \overline{F_{2}}(y)=P\{Y \succ y\}=\exp \left\{-\left(\lambda_{2}+\lambda_{3}\right) y\right\}, y \geq 0
\end{aligned}
$$

Therefore, the marginal distribution of survival of $\mathrm{X}$ and $\mathrm{Y}$ are exponential distribution.

Definition 2.3 If the random variables $(X, Y)$ obey the Marshall-Olkin binary exponential distribution model with parameter $\lambda_{1}, \lambda_{2}$ and $\lambda_{3}$, then the joint probability density function of $X$ and $Y$ is [13]

$$
f(x, y)=\left\{\begin{array}{l}
\lambda_{1}\left(\lambda_{2}+\lambda_{3}\right) \exp \left\{-\lambda_{1} x-\left(\lambda_{2}+\lambda_{3}\right) y\right\}, x \prec y \\
\lambda_{2}\left(\lambda_{1}+\lambda_{3}\right) \exp \left\{-\lambda_{2} y-\left(\lambda_{1}+\lambda_{3}\right) x\right\}, x \succ y \\
\lambda_{3} \exp \left\{-\left(\lambda_{1}+\lambda_{2}+\lambda_{3}\right) y\right\}, x=y
\end{array}\right.
$$

\section{Text}

Taking the fuzzy factors of the non-independent random variables $X$ and $Y$ be $\mu_{1}$ and $\mu_{2}$ respectively, it can set the fuzzy factor as a function of $\mu_{1}$ and $\mu_{2}$, that is $f\left(\mu_{1}, \mu_{2}\right)$ for their joint distribution.

Theorem 1 When random variables $(X, Y)$ obey the Marshall-Olkin binary exponential distribution model with parameters $\lambda_{1}, \lambda_{2}$ and $\lambda_{3}$, then the fuzzy joint reliability function of $X$ and $Y$ is

$$
\bar{\sim}(x, y)=\mu \bar{F}(x, y)=f\left(\mu_{1}, \mu_{2}\right) \exp \left\{-\left[\lambda_{1} x+\lambda_{2} y+\lambda_{3} \max (x, y)\right]\right\}
$$

Proof: Since the fuzzy factor $\mu$ of the joint distribution of $X, Y$ is independent of $X, Y$, and $\mu$ is not a random variable. So it can be assumed that $\mu=f\left(\mu_{1}, \mu_{2}\right)$. According to the relationship between general reliability function $\bar{F}(t)$ and fuzzy reliability function $\bar{F}(\mathrm{t})[14]$, we can know that $\bar{F}(\mathrm{t})=\mu \bar{F}(t)$, so the fuzzy joint reliability function of $X$ and $Y$ can be known.
Theorem 2 The random variable $(X, Y)$ obeys the Marshall-Olkin binary exponential distribution model with parameters $\lambda_{1}, \lambda_{2}$ and $\lambda_{3}$, then the fuzzy joint distribution function of $X$ and $Y$ is that

$$
\underset{\sim}{F}(\mathrm{x}, y)=1-\mu_{1} \overline{F_{1}}(x)-\mu_{2} \overline{F_{2}}(y)+f\left(\mu_{1}, \mu_{2}\right) \bar{F}(x, y)
$$

Proof: When $(X, Y) \sim B V E\left(\lambda_{1}, \lambda_{2}, \lambda_{3}\right)$, the joint distribution function [15] of $X, Y$ is that

$$
F(x, y)=P\{X \prec x, Y \prec y\}=1-\overline{F_{1}}(x)-\overline{F_{2}}(y)+\bar{F}(x, y) \quad,
$$
then the expression of $F(\mathrm{x}, \mathrm{y})=\mu \mathrm{F}(\mathrm{x}, \mathrm{y})$ can be obtained by fuzzy, which represents $\overline{F_{1}}(\mathrm{x}), \overline{F_{2}}(\mathrm{y}), \bar{F}(\mathrm{x}, \mathrm{y})$ were blurred separately.

It $\quad$ is $\quad \overline{F_{1}} \quad$ known that $\overline{F_{1}}(\mathrm{x})=\mu_{1} \overline{F_{1}}(\mathrm{x}), \overline{F_{2}}(\mathrm{y})=\mu_{2} \overline{F_{2}}(\mathrm{y}), \bar{F}(\mathrm{x}, \mathrm{y})=f\left(\mu_{1}, \mu_{2}\right) \bar{F}(\mathrm{x}, \mathrm{y})$, the type (5) can be got finally.

Theorem 3 The random variable $(X, Y)$ obeys the Marshall-Olkin binary exponential distribution model with parameters $\lambda_{1}, \lambda_{2}$ and $\lambda_{3}$, then the fuzzy joint density function of $X$ and $Y$ is $\partial^{2} F /(\partial X \partial Y)$, which is

$$
\underset{\sim}{f(x, y)}=\left\{\begin{array}{l}
f\left(\mu_{1}, \mu_{2}\right) \lambda_{1}\left(\lambda_{2}+\lambda_{3}\right) \exp \left\{-\lambda_{1} x-\left(\lambda_{2}+\lambda_{3}\right) y\right\}, x \prec y \\
f\left(\mu_{1}, \mu_{2}\right) \lambda_{2}\left(\lambda_{1}+\lambda_{3}\right) \exp \left\{-\lambda_{2} y-\left(\lambda_{1}+\lambda_{3}\right) x\right\}, x \succ y \\
f\left(\mu_{1}, \mu_{2}\right) \lambda_{3} \exp \left\{-\left(\lambda_{1}+\lambda_{2}+\lambda_{3}\right) y\right\}, x=y
\end{array}\right.
$$

Theorem 4 The fuzzy reliability function of $Z=\alpha X+\beta Y$ is

$$
\underset{\sim}{\bar{F}}(\mathrm{z})=\mu \bar{F}(\mathrm{z})=f\left(\mu_{1}, \mu_{2}\right) \bar{F}(z)
$$

Proof: Given the joint density function of $\mathrm{X}$ and $\mathrm{Y}$, the probability density function of $Z=\alpha X+\beta Y$ can be obtained by using the Jacobian determinant.

Assume $\left\{\begin{array}{l}Z=\alpha X+\beta Y \\ U=\beta Y\end{array}\right.$, deformation can be obtained $\left\{\begin{array}{l}X=\frac{Z-U}{\alpha} \\ Y=\frac{U}{\beta}\end{array}\right.$

Because $X, Y \succ 0$ and $0 \prec u \prec z$, Jacobian determinant is $\frac{1}{\alpha \beta}$ by calculating, so the joint density function of $Z$ and $\mathrm{U}$ is 


$$
P(z, u)=\frac{1}{\alpha \beta} P\left(\frac{z-u}{\alpha}, \frac{u}{\beta}\right)=\left\{\begin{array}{l}
\frac{f\left(\mu_{1}, \mu_{2}\right) \lambda_{2}\left(\lambda_{1}+\lambda_{3}\right)}{\alpha \beta} \exp \left\{-\left[\lambda_{2} \frac{u}{\beta}+\left(\lambda_{1}+\lambda_{3}\right) \frac{z-u}{\alpha}\right]\right\}, u \prec \frac{\beta z}{\alpha+\beta} \\
f\left(\mu_{1}, \mu_{2}\right) \lambda_{3} \exp \left\{-\left(\lambda_{1}+\lambda_{2}+\lambda_{3}\right) \frac{u}{\beta}\right\}, u=\frac{\beta z}{\alpha+\beta} \\
\frac{f\left(\mu_{1}, \mu_{2}\right) \lambda_{1}\left(\lambda_{2}+\lambda_{3}\right)}{\alpha \beta} \exp \left\{-\left[\lambda_{1} \frac{z-u}{\alpha}+\left(\lambda_{2}+\lambda_{3}\right) \frac{u}{\beta}\right]\right\}, u \succ \frac{\beta z}{\alpha+\beta}
\end{array}\right.
$$

Thus, the marginal density function of $Z$ is

$$
\begin{aligned}
& \mathrm{p}_{z}(z)=\int_{0}^{z} p(z, u) d u \\
& =\frac{f\left(\mu_{1}, \mu_{2}\right) \lambda_{2}\left(\lambda_{1}+\lambda_{3}\right)}{\alpha \beta} \int_{0}^{\frac{\beta z}{\alpha+\beta}} \exp \left\{-\left[\lambda_{2} \frac{u}{\beta}+\left(\lambda_{1}+\lambda_{3}\right) \frac{z-u}{\alpha}\right]\right\} d u \\
& +\frac{f\left(\mu_{1}, \mu_{2}\right) \lambda_{1}\left(\lambda_{2}+\lambda_{3}\right)}{\alpha \beta} \int_{\frac{\beta z}{\alpha+\beta}}^{z} \exp \left\{-\left[\lambda_{1} \frac{z-u}{\alpha}+\left(\lambda_{2}+\lambda_{3}\right) \frac{u}{\beta}\right]\right\} d u \\
& \bar{F}(\mathrm{z})=\mathrm{P}(\mathrm{Z} \succ \mathrm{z}) \\
& =\frac{f\left(\mu_{1}, \mu_{2}\right) \lambda_{2}\left(\lambda_{1}+\lambda_{3}\right)}{\beta\left(\lambda_{1}+\lambda_{3}\right)-\alpha \lambda_{2}} \int_{z}^{\infty}\left\{\exp \left(-\frac{\lambda_{1}+\lambda_{2}+\lambda_{3}}{\alpha+\beta} z\right)-\exp \left(-\frac{\lambda_{1}+\lambda_{3}}{\alpha} z\right)\right\} d z \\
& -\frac{f\left(\mu_{1}, \mu_{2}\right) \lambda_{1}\left(\lambda_{2}+\lambda_{3}\right)}{\beta \lambda_{1}-\alpha\left(\lambda_{2}+\lambda_{3}\right)} \int_{z}^{\infty}\left\{\exp \left[-\left(\frac{\lambda_{1}+\lambda_{2}+\lambda_{3}}{\alpha+\beta}\right)\right]-\exp \left[-\left(\frac{\lambda_{2}+\lambda_{3}}{\beta} z\right)\right]\right\} d z
\end{aligned}
$$

$$
\begin{aligned}
& =\frac{1}{\alpha \beta} \frac{f\left(\mu_{1}, \mu_{2}\right) \lambda_{2}\left(\lambda_{1}+\lambda_{3}\right)}{\frac{1}{\alpha}\left(\lambda_{1}+\lambda_{3}\right)-\frac{1}{\beta} \lambda_{2}}\left\{\exp \left[-\frac{\lambda_{2}}{\alpha+\beta} z-\frac{\lambda_{1}+\lambda_{3}}{\alpha+\beta} z\right]-\exp \left[-\frac{1}{\alpha}\left(\lambda_{1}+\lambda_{3}\right) z\right]\right\} \\
& -\frac{1}{\alpha \beta} \frac{f\left(\mu_{1}, \mu_{2}\right) \lambda_{1}\left(\lambda_{2}+\lambda_{3}\right)}{\frac{1}{\alpha} \lambda_{1}-\frac{1}{\beta}\left(\lambda_{2}+\lambda_{3}\right)}\left\{\exp \left[-\frac{\lambda_{1}}{\alpha+\beta} z-\frac{\lambda_{2}+\lambda_{3}}{\alpha+\beta} z\right]-\exp \left[-\frac{\lambda_{2}+\lambda_{3}}{\beta} z\right]\right\}
\end{aligned}
$$

It can be seen that the fuzzy reliability function of $Z$ is

Finishing can get

$$
\begin{aligned}
& \bar{\sim}(z)=\frac{f\left(\mu_{1}, \mu_{2}\right) \lambda_{2}\left(\lambda_{1}+\lambda_{3}\right)}{\alpha \lambda_{2}-\beta\left(\lambda_{1}+\lambda_{3}\right)}\left[\frac{\alpha}{\lambda_{1}+\lambda_{3}} \exp \left(-\frac{\lambda_{1}+\lambda_{3}}{\alpha} z\right)-\frac{\alpha+\beta}{\lambda_{1}+\lambda_{2}+\lambda_{3}} \exp \left(-\frac{\lambda_{1}+\lambda_{2}+\lambda_{3}}{\alpha+\beta} z\right)\right] \\
& +\frac{f\left(\mu_{1}, \mu_{2}\right) \lambda_{1}\left(\lambda_{2}+\lambda_{3}\right)}{\alpha\left(\lambda_{2}+\lambda_{3}\right)-\beta \lambda_{1}}\left[\frac{\alpha+\beta}{\lambda_{1}+\lambda_{2}+\lambda_{3}} \exp \left(-\frac{\lambda_{1}+\lambda_{2}+\lambda_{3}}{\alpha+\beta} z\right)-\frac{\beta}{\lambda_{2}+\lambda_{3}} \exp \left(-\frac{\lambda_{2}+\lambda_{3}}{\beta} z\right)\right]
\end{aligned}
$$

Reliability function [16] of $Z=\alpha X+\beta Y$ is that

$$
\begin{aligned}
& \bar{F}(z)=\frac{\lambda_{2}\left(\lambda_{1}+\lambda_{3}\right)}{\alpha \lambda_{2}-\beta\left(\lambda_{1}+\lambda_{3}\right)}\left[\frac{\alpha}{\lambda_{1}+\lambda_{3}} \exp \left(-\frac{\lambda_{1}+\lambda_{3}}{\alpha} z\right)-\frac{\alpha+\beta}{\lambda_{1}+\lambda_{2}+\lambda_{3}} \exp \left(-\frac{\lambda_{1}+\lambda_{2}+\lambda_{3}}{\alpha+\beta} z\right)\right] \\
& +\frac{\lambda_{1}\left(\lambda_{2}+\lambda_{3}\right)}{\alpha\left(\lambda_{2}+\lambda_{3}\right)-\beta \lambda_{1}}\left[\frac{\alpha+\beta}{\lambda_{1}+\lambda_{2}+\lambda_{3}} \exp \left(-\frac{\lambda_{1}+\lambda_{2}+\lambda_{3}}{\alpha+\beta} z\right)-\frac{\beta}{\lambda_{2}+\lambda_{3}} \exp \left(-\frac{\lambda_{2}+\lambda_{3}}{\beta} z\right)\right]
\end{aligned}
$$

$$
\text { So } \bar{F}(\mathrm{z})=\mu \bar{F}(z)=f\left(\mu_{1}, \mu_{2}\right) \bar{F}(z)
$$

\section{Conclusion}

Exponential distribution is one of the commonly used distributions in reliability analysis [17]. With the development of research, people found many practical engineering problems not only exist randomness, but also have a wide range of fuzziness [18]. Therefore, the fuzzy theory is introduced into the research of reliability, the research of fuzzy reliability is carried out [19]. In recent years, a variety of binary index distribution has also been proposed, the model Marshall-Olkin is a very useful binary exponential distribution model [20]. In the study of reliability function, it is known that the fuzzy reliability functions of the general variables under relatively independent conditions and the reliability functions of the two-dimensional variables under non-independent conditions are all completed. Therefore, based on the Marshall-Olkin binary exponential distribution model, this paper gives the fuzzy joint reliability function of $X$ and $Y$, the fuzzy joint distribution function of $X$ and $Y$, the fuzzy joint density function of $X$ and $Y$ and the fuzzy reliability function of $Z=\alpha X+\beta Y$ in the case of $X, Y$ non - independent, which is of great 
significance to practice. Of course, we will discuss the distribution of $Z=\alpha X+\beta Y$ when $X, Y$ subject to other types of distribution in the future work.

\section{References}

[1] Qiao Aifang. Generalized exponential distribution parameter change point analysis and its application [D]. Qinghai Normal University, 2016.

[2] Liu Guoxiang. Relationship between exponential distribution and other distributions [J]. Journal of Chifeng University (Natural Science Edition), 2011, (12): 12-14.

[3] Zeng Tianxiang. Application and Limitation of Exponential Distribution [J]. Actuation of Aviation, 1983, (06): 14-20.

[4] Xi Li, Liu Ruiyuan. Two-dimensional exponential distribution [J] Qinghai University (Natural Science), 2007, (02): 86-88 + 94.

[5] Zhang Ping. Statistical Analysis of Bivariate Exponential Distribution [D]. Shanghai Normal University, 2011.

[6] Han Zhu. Research on Characterization and Parameter Estimation of Bivariate Exponential Distributions and Other Related Problems [D]. Ningbo University, 2009.

[7] Arjun K,Gupta and SaraleesNadarajah. Exact and approximate distributions for the linear combination of inverted Dirichlet components [J]. Journal of Japan Statistical Society, 2006, 36 (2): $225-236$.

[8] Li Dongna, Zhang Minyue, Ma Changqing. Fuzzy reliability analysis of parallel series system [J]. Journal of Gansu science, 2007, (04): 141-144.

[9] Li Tingjie, Gao He. Fuzzy Reliability of Series System [J]. Fuzzy Systems and Mathematics, 1989, (01): 38-45.

[10] GuoYunfei, Yin Zhe. The distribution of linear combinations of non - independent random variables subjecting to two- dimensional exponential distribution [J]. Mathematical Practice and Understanding, 2010, 40 (16): 179-183.

[11] Zhou Juling, Liang Xiaojia. Marshall-Olkin binary exponential distribution [J]. Journal of Xinjiang Normal University (Natural Science Edition), 2013, (04): 63-65.

[12] Li Guoan. Independence and Uniqueness of Exponential Distribution of Binary Marshall Olkin Type [J]. University Mathematics, 2013,29 (4): 91-93. DOI: 10.3969/ j.issn.1672-1454.2013.04.019.

[13] Saralees Narajah and Samuel Kotz. Reliability for some Bivariate exponential distributions, Mathematical Problems in Engineering, 1-14, 2006.

[14] LI Tingjie, Gao He. Fuzzy Reliability [J]. Systems Engineering and Electronics, 1988, (10): 1-9.

[15] Cheng Kan, Cao Jinhua, Introduction to Reliability Mathematics. Beijing: Higher Education Press, 2006.

[16] Guo Yunfei, Reliability function of linear combination of marshall and olkin's bivariate exponential distribution, Scientific Advances Publishers, 2015.

[17] Li Guoan. Exponential Distribution Sampling Fundamental Theorem and Its Application in Parameter Estimation of Two-parameter Binary Marshall-Olkin Exponential Distribution Parameter [J]. Statistical Research, 2016, (07): 98-102.

[18] Wang Junfang, Zhang Minyue. Fuzzy reliability of [J]. Journal of Sichuan University of Science and Engineering two interdependent parts in parallel system and series system with cold standby components (NATURAL SCIENCE EDITION), 2015, (01): 80-82.

[19] Li Tingjie, GaoHe. Reliability Design [M]. Beijing: Beijing University of Aeronautics and Astronautics Press, 1988.

[20] Li Guoan. Characteristic and Parameter Estimation of Multivariate Marshall-Olkin Exponential Distribution [J]. Journal of Engineering Mathematics, 2005, (06): 109-116. 